ACTA UIVERSITATIS CIBINIENSIS - TECHNICAL SERIES

Vol. LXVIII 2016

\title{
ABOUT THE SMART WEATHER STATION
}

\author{
BOGDAN Mihai \\ Associate professor, Ph.D, Faculty of Engineering, Computer Science and Electrical Engineering \\ Department, ”Lucian Blaga”University of Sibiu, Romania, mihai.bogdan@ulbsibiu.ro
}

\begin{abstract}
Until recently, the Romanian weather stations utilized ordinary transducers that acquire useful information related to the desired physical inputs. These inputs will be converted into electrical signals easy to be processed by analog to digital converters.

This paper proposed a new approach based on smart sensors system that change the interior behavior in order to optimize data acquirements from the environment. The smart sensor characteristics are stored into himself in a transducer electronic data sheet form (TEDS). The intelligent transducer generat together with the measured analogic signal also a digital interface. Through this interface the transducer's catalog data can be read from the transducer.
\end{abstract}

Key words: smart sensor, UV sensor, Transducer Electronic Data Sheet

\section{Introduction}

Using meteorological satellites to monitor the weather on Earth has its advantages and disadvantages. Images from space gives opportunity to observe any point on the planet, and extraction of information, despite the restrictions imposed by the borders of a state, or access difficult conditions there. Getting the same data by conventional methods require a large number of weather stations (which are expensive) under the most inhospitable places. Despite this advantage, the satellites are not able to provide the same accuracy class as conventional instruments that are in a meteorological station, being useful only for providing information about regional and global environment.

A weather station is essentially a data acquisition system remotely able to collect information on temperature, sunlight, humidity, wind speed and wind direction in a given geographical area. These data are acquired using different types of sensors and then sent to a microcontroller that processes the data by making them available. The data is then either stored or transmitted. The system is equipped with two stations: a base station and a remote station. The block diagram of the base station it is presented in Figure 1 and the block diagram of the remote station, in Figure 2. Both the base station and remote station has a transmitter, a receiver and a microcontroller. The role of the microcontroller is to convert analog and digital signals and to manipulating the control signals, to and from the base station.

Powered base station is made from a 12 volt battery, and the remote station is made from a solar panel. Sensors for data collection are:

- a sensor for measuring the temperature, for example, the Analog Devices AD592 sensor, which has the advantage of an linear output signal (current) proportional to the temperature;

- a ultraviolet light sensor (UV). That must follow the sun using a photo sensor and a stepper motor;

- one single sensor both for visible light (VIS) as well as infrared light (IR);

- an moisture sensor, for example SC-600 sensor of the Ohmic Instruments corporation;

- an anemometer for measuring wind speed and a vane for indicating wind direction

Communication with the remote system will be conducted using transmitters and the radio frequency receivers. 


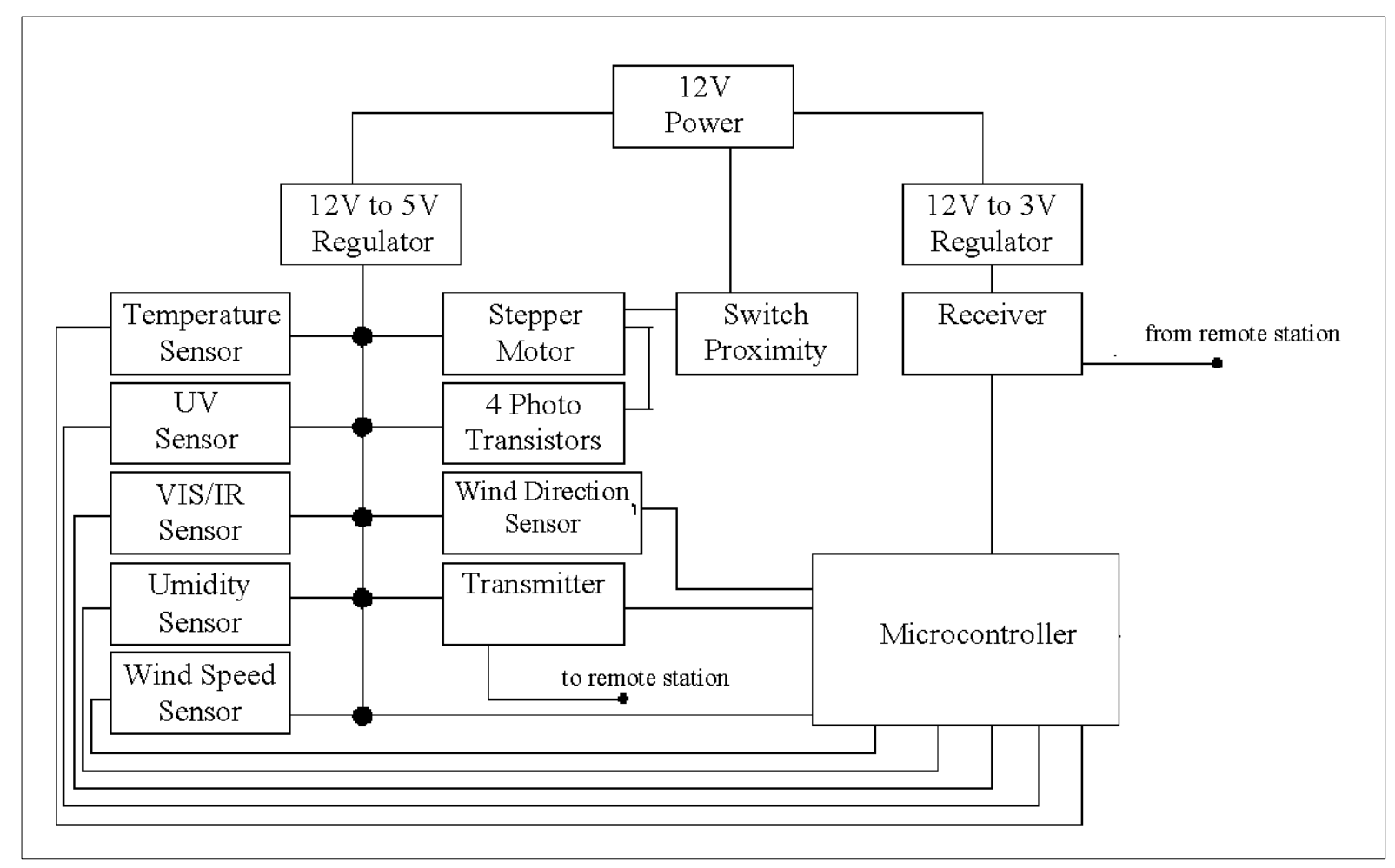

Figure 1. Block diagram of the base station

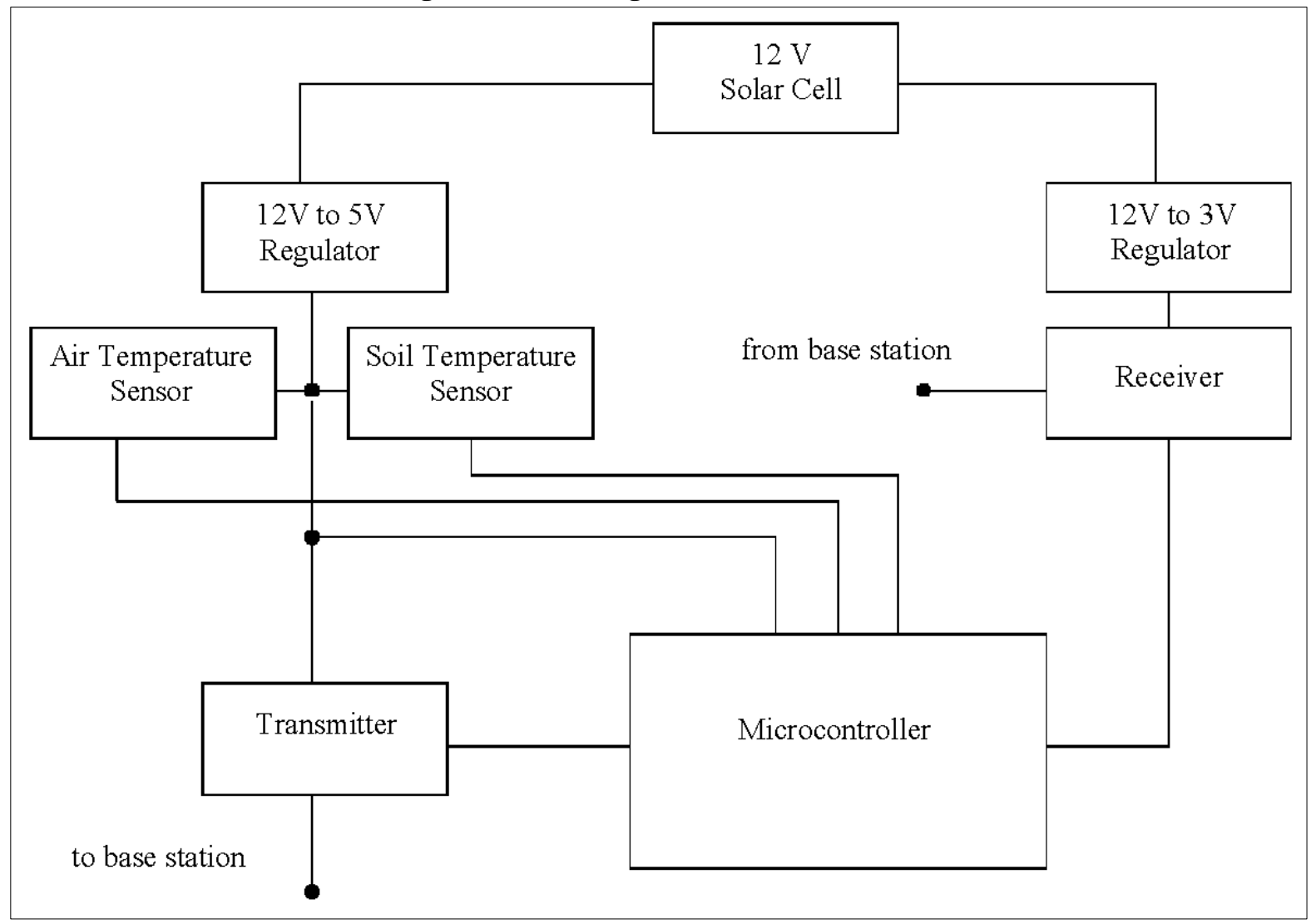

Figure 2. The remote station block diagram

\section{Smart Sensors}

The integration of microelectronics and micro-machines led to a revolution in control systems through the development of micro electromechanical systems (MEMS). MEMS enables the integration of 
micromechanical structures, sensors and signal conditioning circuits into a single module. Recent advantages of integrated circuits allow embedding computing capabilities, such as DSPs and microcontroller with an integrated sensor module in a single chip [1].

The indisputable advantages of microelectronics led to implementation of new tests in the physical construction of integrated sensors, such as: MEMS (Micro-Electrical-Mechanical Systems) and SoC (Systems-on-Chip).

Microsystem technology (MST) offers new ways of combining elements sensitive to processing and communication circuitry, on the same chip [2].

Lately, intelligent sensors have gained widespread in various industrial fields, especially in the composition of complex automation systems, robots, etc. The most common way of obtaining information on physical phenomena is based on the evaluation of voltage or current amplitude obtained from the sensor.

Smart sensor is a chip without external components that include sensitive elements for capture the physical signal, that have analog and digital processing of the signal received from them but also intelligent functions: self-testing, self-identification, self-validation or self-adaptive

Incorporating microprocessor inside sensors, by microelectronics technology, allows multiple functionalities thereof. Sensor manufacturers are looking for ways to reduce cost price and their manufacturing, smart sensors systems covering a continuous demand for more and more sophisticated applications. Sensor networks have penetrated into every industry, being easy to use, causing a major change in measurement techniques [4].

Intelligent sensors have a number of advantages in terms of cost price and in terms of complexity:

- Sensor electrical signals rough and nonlinear, can be conditioning, calibrated and converted to digital format by a single chip, eliminating external noise transmission lines;

- Smart Sensor is equipped with a digital communication interface, providing capability "plug-andplay" containing a electronic data table, stored electronically in the memory of the sensor;

- With smart sensors it is possible to monitor the local system via a communication network, thus simplifying system diagnostics. transducers.

The critical issue in designing applications with smart transducer is the construction of these interfaces

\section{Interfaces for inteligence sensors}

The interfacing of a process is defined as a functional unit that adapts equipment for process control with the computing system associated with it [3].

The objectives of this standard is:

- simplification and ease of the integration of sensors into applications;

- defining a common interface for the sensing element;

- making a simple interface for transducers independent networks;

They are set standardized connection methods for transmitters smart control networks using existing technology. So far there are five standards:

- 1451.1 standard, it is an independent network model and defines the network model with application processor (NCAP) and NCAP model adapted to different networks

- 1451.2 standard, it is for smart sensors and defines the interface for smart sensor module (STIM), defines electronic data sheet of the transducer (TEDS) and defines the transducer interface (TII) with the specifications communication protocol between STIM and NCAP.

- 1451.3 standard, propose a digital standard interface (TBIM) able to connect multiple transducers in a multi-drop configuration.

- 1451.4 standard, it proposes a standard interface that allows analog transducers operate in a mixed signal (analog / digital). This standard establishes a universally accepted method for plug-and-play capability of the sensors.

- 1451.5 standard, defines an interface for wireless communication (radio). 


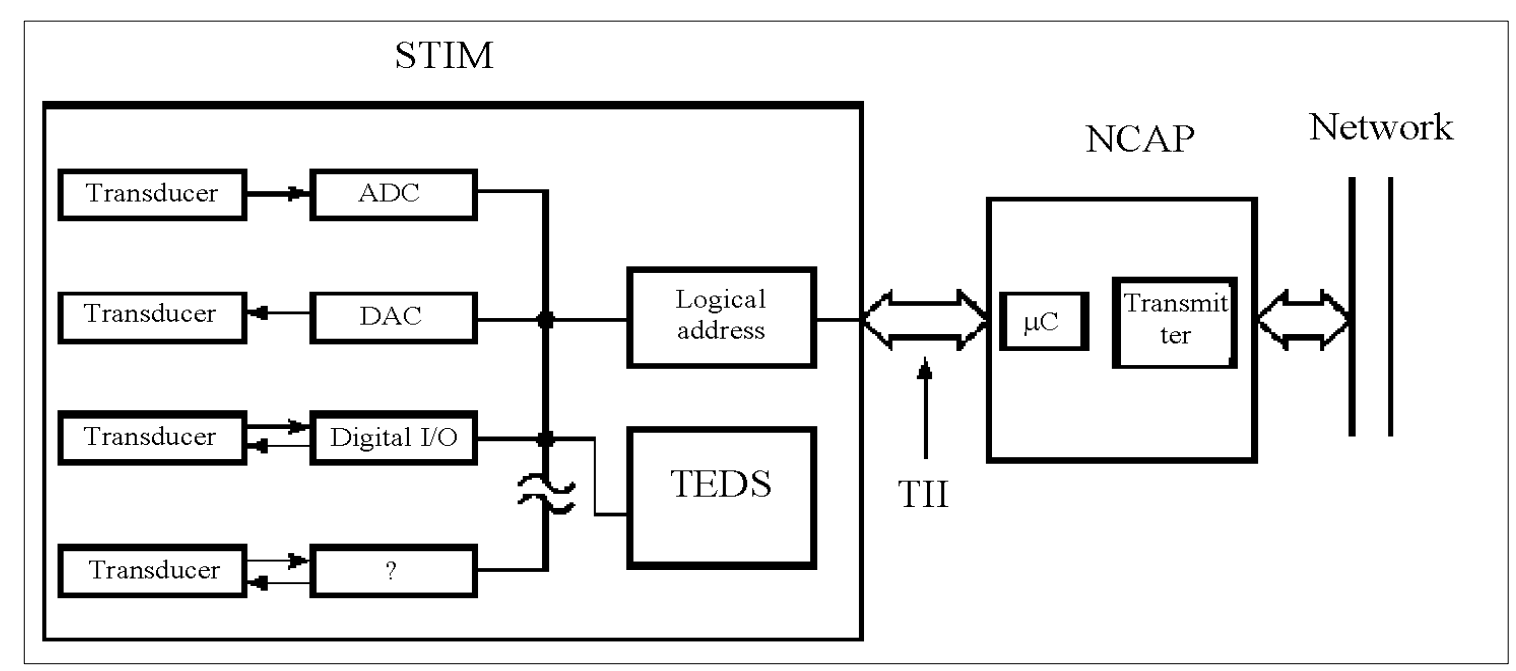

Figure 3. 1451.2 standard structure

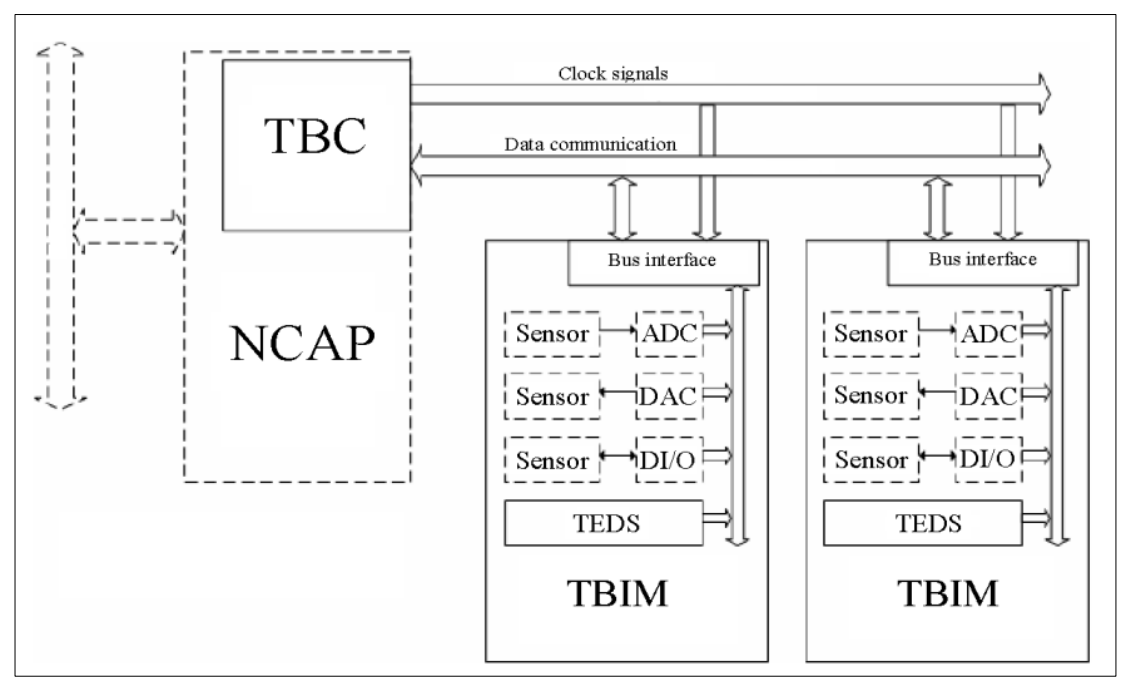

Figure 4. 1451.3 standard structure

\section{Conclusions}

This paper proposes a remote data acquisition system for acquisition of various weather quantity such as air temperature, soil temperature, water temperature, wind direction, wind speed and air humidity. Are presented various standards for smart sensors interfacing to microprocessor based systems or to various bus network. Intelligent sensors have a number of advantages in terms of technology, cost and management complexity compared to ordinary sensors.

1451 standard also offers features "plug and play" for connecting sensors at various bus fields and at various existing network.

\section{References}

1. Sergey Y. Yurish, Smart Sensors Systems Design, International Frequency Sensor Association (IFSA), www.sensorsportal.com

2. N. V. Kirianaki, S.Y. Yurish, N.O. Shpak, V.P. Deynega, Data Acquisition and Signal Processing for Smart Sensors, 1st Edition, Jon Wiley\&Sons Ltd., 2002

3. Gerard C.M. Meijer, Smart Sensor Systems, John Wiley \& Sons, Ltd. ISBN: 978-0-470-86691-7, 2008

4. Randy Frank, Understanding Smart Sensors, Second Edition, ISBN 0-89006-311-7, ARTECH HOUSE, INC., 2000

5. Waldemar Nawrocki, Measurement Systems and Sensors, ISBN 1-58053-945-9, ARTECH HOUSE, INC., 2005

6. http://grouper.ieee.org/ 